OPEN ACCESS

Investigation of Purcell Factor for an Activator Ion due to Surface Plasmon Modes

To cite this article: J. Collins and K. C. Mishra 2020 ECS J. Solid State Sci. Technol. 9016002

View the article online for updates and enhancements. 


\title{
Investigation of Purcell Factor for an Activator Ion due to Surface Plasmon Modes
}

\author{
J. Collins $\circledast^{1, *, \mathrm{z}}$ and K. C. Mishra $\oplus^{2, * *}$ \\ ${ }^{1}$ Department of Physics and Astronomy, Wheaton College, Norton, Massachusetts 02766, USA \\ ${ }^{2}$ North Chelmsford, Massachusetts 01863, USA
}

\begin{abstract}
We considered earlier the role of the surface plasmon states in the spontaneous decay of an optically active ion in proximity to a flat, metallic surface. ${ }^{1}$ In this work we have extended our earlier results to obtain an expression for the density of plasmon states accessible to the ion. We express the spontaneous decay rate of the ion into the surface plasmon field in terms of the density of final states and the appropriate "Einstein" B-coefficient, which depends on the matrix elements of the ion-surface plasmon interaction. Next we derive an expression for the contribution of the surface plasmons to the total radiative decay rate, and determine the Purcell factor of this system. Finally, calculations are presented showing how the Purcell factor for an optically active ion near a silver surface depends on the wavelength of the transition and distance of the ion from the surface.

(C) The Author(s) 2019. Published by ECS. This is an open access article distributed under the terms of the Creative Commons Attribution 4.0 License (CC BY, http://creativecommons.org/licenses/by/4.0/), which permits unrestricted reuse of the work in any medium, provided the original work is properly cited. [DOI: 10.1149/2.0032001JSS]

(cc) BY
\end{abstract}

Manuscript submitted June 14, 2019; revised manuscript received July 12, 2019. Published August 20, 2019. This paper is part of the JSS Focus Issue on Recent Advances in Wide Bandgap III-Nitride Devices and Solid State Lighting: A Tribute to Isamu Akasaki.

The presence of a metallic surface affects the luminescence characteristics of a nearby optically active ion. This feature has been exploited in solid state light sources to extract light form within the LEDs ${ }^{2-11}$ and to extract more photons from both the LED and the phosphor composites $^{12-23}$ in specific configurations. Many lighting laboratories are exploring plasmon-structured LED surfaces and phosphor composites to extract more light in certain directions. In this paper we explore if the presence of a metallic surface near optical ions could indeed enhance the extraction of light from the phosphor composite. One measure of this extraction is the Purcell factor ${ }^{24}$ of the device, which is addressed in this work.

In particular, a metallic surface can influence the spontaneous decay rate of an ion through, for example, redistribution of the photon modes due to the presence of the mirror ${ }^{25,26}$ and the interaction with the surface plasmon-polariton modes of the metal surface. In this work, we consider only the latter effect, that is, the interaction between an optically active ion and the surface plasmon-polariton field associated with the metallic layer, and determine its influence on the decay of an excited ion. For simplicity, in the remainder of the work we shall use the phrase "surface plasmon" instead of "surface plasmon polariton" sometimes used in the literature.

Since the surface plasmons provide an additional set of electromagnetic modes into which the ion might decay, they tend to increase spontaneous decay rate of the ion, thereby decreasing its lifetime. It is possible to express the decay rate of the ion into the surface plasmon modes using an Einstein $B$ coefficient relevant to the ion-surface plasmon interaction. ${ }^{1,28}$ The goals of this paper are to find expressions for the final density of surface plasmon states, the decay rate of the ion due to the surface plasmon modes, and the resulting Purcell factor. Also, we estimate the Purcell factor for the specific case of an ion near a silver metallic surface, and examine in detail the effect of the metallic layer on the emitting ion.

\section{Theory}

The radiative decay rate for spontaneous emission of a photon is given by

$$
\frac{1}{\tau_{\text {rad }}}=B \rho(\nu) h \nu
$$

where $\tau_{\text {rad }}$ is the radiative lifetime of the ion into the photon modes, $B$ is the Einstein $B$ coefficient, $\rho(v)$ is the final density of photon states, and $h \nu$ is the energy of the emitted photon. We note that in Eq. $1 \rho(v)$ has units of number of modes per unit volume per unit frequency.

For an ion radiating into the surface plasmon modes, we make the conjecture that the decay rate is given by an expression similar to Eq. 1,

$$
\frac{1}{\tau_{s p}}=B_{s p} \rho_{s p}(\nu) h \nu
$$

where the subscript "sp" refers to the surface plasmons, and $h v$ the energy of the emitted surface plasmon. Thus, determining the rate at which the ion decays into the surface plasmon modes requires the knowledge of the corresponding "Einstein" $B$ coefficient, $B_{s p}$, as well as the density of surface plasmon modes, $\rho_{s p}(\nu) . \rho_{s p}(\nu)$ must have the same units as the corresponding term in Eq. 1, that is, number of modes per unit volume per unit frequency.

For a smooth planar surface, one can find the density of possible surface modes, $\sigma_{s p}(\nu) .{ }^{1}$ Defining $p$ as the wavevector of the surface waves, it is found that

$$
\sigma_{s p}(\nu)=p \frac{d p}{d \omega}
$$

where the units of $\sigma_{s p}(\nu)$ are the number of modes per unit area per unit frequency. The corresponding dispersion relation for $p$ is

$$
p=\frac{\omega}{c}\left(\frac{\varepsilon_{1} \varepsilon_{2}}{\varepsilon_{1}+\varepsilon_{2}}\right)^{1 / 2} .
$$

where $\varepsilon_{1}$ and $\varepsilon_{2}$ the dielectric constants of the metal and of the adjacent dielectric medium, respectively. The density of surface modes given in Eq. 3 is for a two dimensional, flat surface - the metallic surface on which the plasmons propagate. The field associated with the propagating surface plasmons extends into the dielectric, and so is felt by the optical ion. We are interested in the density of modes available at the site of the ion, which should decrease as the ion-surface distance decreases, and should have the appropriate units of modes per unit volume per unit frequency. Thus, we propose the following form for the density of surface plasmon modes,

$$
\rho_{s p}(\nu)=\frac{\sigma_{s p}(\nu)}{L(\omega, d)}
$$

where $L(\omega, d)$ is a length parameter, ${ }^{1,27}$ which depends on both the surface plasmon frequency, $\omega$, and on the distance, $d$, between the ion and the metallic surface. $L(\omega, d)$, introduced in Eq. 5 makes the units of $\rho_{s p}(\nu)$ consistent with the density of states term in Eq. 2 .

For the length parameter, we will use ${ }^{1}$

$$
L(\omega, d)=\frac{\omega^{3}}{2 c^{2}} \frac{\varepsilon_{2}^{3 / 2}\left(\varepsilon_{2}-\varepsilon_{1}\right)}{\left(-\varepsilon_{1}\right)^{1 / 2}} \frac{1}{p^{4}} \frac{d p}{d \omega} e^{2 \alpha_{2} d} .
$$


In Eq. 7, $d$ is the distance of the ion from the metallic surface and $\alpha_{2}$ is given by

$$
\alpha_{2}=i \frac{\omega}{c} \frac{\varepsilon_{2}}{\left(\varepsilon_{1}+\varepsilon_{2}\right)^{1 / 2}}
$$

In the frequency range of interest, $\varepsilon_{1}$ is negative and $\left|\varepsilon_{1}\right|>\varepsilon_{2}$, so that $\varepsilon_{1}+\varepsilon_{2}<0$ and $\alpha_{2}$ is real. Inserting Eqs. 3, 4, and 6 into Eq. 5, we obtain the following expression for the density of surface plasmon modes at the site of the ion.

$$
\rho_{s p}(\nu)=\frac{8 \pi^{2}}{c^{3}} \nu^{2}\left[\frac{\left(-\varepsilon_{1}\right)^{5 / 2}\left(\varepsilon_{1}\right)^{1 / 2} \varepsilon_{2}}{\left(\varepsilon_{1}+\varepsilon_{2}\right)^{5 / 2}\left(\varepsilon_{2}-\varepsilon_{1}\right)}\right] e^{-2 \alpha_{2} d} .
$$

In Eq. 8 the density of states decreases exponentially as the ion-metallic surface distance increases. $\rho_{s p}(\nu)$ has as explicit dependence on frequency and also an implicit dependence on frequency, mainly through $\varepsilon_{1}$. Finally, the term in front of the brackets in Eq. 8 is simply the density of photon states for the ion in vacuum in the absence of the metal surface.

In order to calculate the spontaneous decay rate into the surface plasmon modes, we need the Einstein B coefficient, which contains the matrix elements that drive the transitions. A form the Einstein B coefficient has been developed by Mishra et al. using a quantum electrodynamic approach, and is given by ${ }^{1}$

$$
B_{s p}=\frac{2 \pi}{\hbar^{2}}\left(\mu_{\perp}^{2}-\frac{\varepsilon_{2}}{2 \varepsilon_{1}} \mu_{\|}^{2}\right) .
$$

To find the decay rate of the ion into the surface plasmon modes, we insert Eq. 8 into Eq. 2 to obtain

$$
\frac{1}{\tau_{s p}}=B_{s p} h \nu\left(\frac{8 \pi^{2}}{c^{3}} \nu^{2}\right)\left[\frac{\left(-\varepsilon_{1}\right)^{5 / 2}\left(\varepsilon_{1}\right)^{1 / 2} \varepsilon_{2}}{\left(\varepsilon_{1}+\varepsilon_{2}\right)^{5 / 2}\left(\varepsilon_{2}-\varepsilon_{1}\right)}\right] e^{-2 \alpha_{2} d} .
$$

where $B_{s p}$ is given in Eq. 9. The total decay rate of the optical ion into electromagnetic modes includes the decay rate into the vacuum photon modes (Eq. 1) plus the contribution of the decay into surface plasmon modes (Eq. 10).

\section{Results and Discussion}

Case 1: dipole perpendicular to metal surface.-Let us first examine the case when the dipole moment of the ion is oriented perpendicular to the metallic surface. In this case the Einstein $B$ coefficients corresponding to the decay via photons and surface plasmons are identical, that is,

$$
B=B_{s p}=\frac{2 \pi}{\hbar^{2}}\left(\mu_{\perp}^{2}\right) .
$$
by

The total decay rate of the ion into electromagnetic modes is given

$$
\begin{aligned}
\frac{1}{\tau_{\text {rad }}}+\frac{1}{\tau_{s p}} & =B \rho(\nu) h \nu+B_{s p}(\nu) \rho_{s p}(\nu) h \nu \\
& =B h \nu \frac{8 \pi^{2}}{c^{3}} \nu^{2}\left[1+f\left(\varepsilon_{1}, \varepsilon_{2}\right) e^{-2 \alpha_{2} d}\right]
\end{aligned}
$$

where

$$
f\left(\varepsilon_{1}, \varepsilon_{2}\right)=\left[\frac{\left(-\varepsilon_{1}\right)^{5 / 2}\left(\varepsilon_{1}\right)^{1 / 2} \varepsilon_{2}}{\left(\varepsilon_{1}+\varepsilon_{2}\right)^{5 / 2}\left(\varepsilon_{2}-\varepsilon_{1}\right)}\right]
$$

The Purcell factor $F_{\perp}$, which gives the fractional increase of the spontaneous decay rate due to an ion with its dipole oriented perpendicular to the surface into the surface plasmon modes is given by

$$
F_{\perp}=\frac{\left(\frac{1}{\tau_{r a d}}+\frac{1}{\tau_{s p}}\right)}{\frac{1}{\tau_{r a d}}}=1+\left(\frac{\frac{1}{\tau_{s p}}}{\frac{1}{\tau_{r a d}}}\right)=1+f\left(\varepsilon_{1}, \varepsilon_{2}\right) e^{-2 \alpha_{2} d} .
$$

Thus, contribution of the decay of the ion due to the surface plasmon modes relative to the contribution of the photon modes is given by the term $f\left(\varepsilon_{1}, \varepsilon_{2}\right) e^{-2 \alpha_{2} d}$. We note that the above Purcell factor ignores

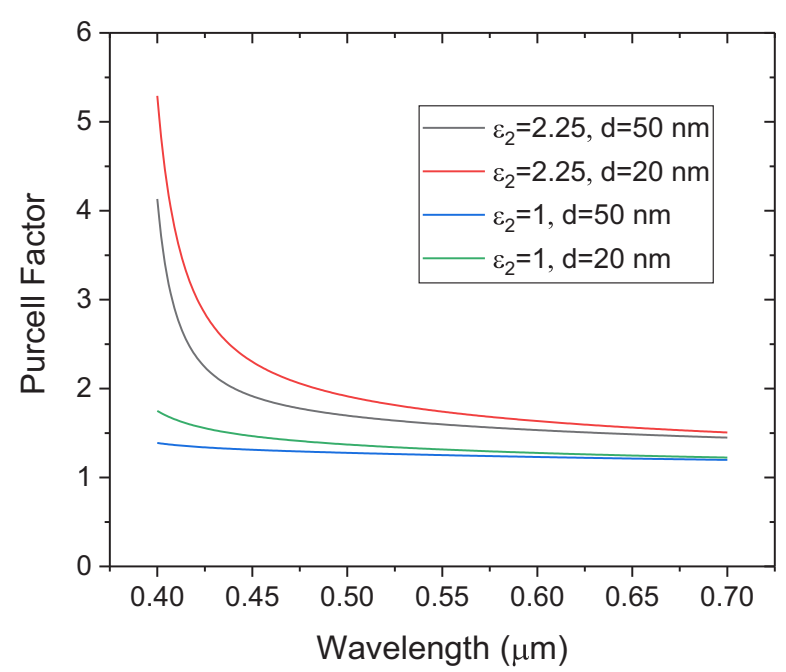

Figure 1. Purcell factors vs. wavelength for $\varepsilon_{2}$ of 2.25 and 1.0 and distances (d) from the silver surface of 20 and $50 \mathrm{~nm}$.

the Drexhage effect, ${ }^{1,25,26}$ and only includes the effect of the surface plasmons.

Figure 1 shows the behavior of the Purcell factor as a function wavelength for the ion-surface distances, $d$, of $20 \mathrm{~nm}$ and $50 \mathrm{~nm}$, and for the cases when $\varepsilon_{2}$ is set equal to 1.0 and to 2.25. In Figure 1, we have assumed a silver layer, so that $\varepsilon_{1}$ varies roughly between -20 and -3 moving from the red to violet ends of the visible spectrum. ${ }^{29} \mathrm{We}$ note the following trends:

(1) The contribution of the surface plasmon modes to the decay rate of the ion increases as wavelength decreases in the visible region.

(2) The decay of the ion due to surface plasmons modes decreases exponentially as the ion-metal distance decreases.

(3) As the dielectric constant of the material in which the ion is located increases from 1.0 (vacuum) to 2.25 (typical polymers or glass), the ion to surface plasmon decay rate increases.

In Eq. 10 , the term $\left(\varepsilon_{1}+\varepsilon_{2}\right)$ in the denominator approaches zero as $\varepsilon_{2}$ approaches $-\varepsilon_{1}$. This resonance condition is achieved in the case of silver at wavelengths slightly less than $400 \mathrm{~nm}$ for a typical oxide with refractive index near 1.7. In deriving Eq. 10, we assumed that $\varepsilon_{1}$ is negative and $\left|\varepsilon_{1}\right|>\varepsilon_{2}$, so that $\varepsilon_{1}+\varepsilon_{2}<0$. Therefore, we only examined values of the refractive index between 1 and 1.5, i.e., for $1<\varepsilon_{2}<2.25$. Some representative data from Figure 1 are also shown in Table I.

Case 2: average orientation of dipole with respect to metal surface.-For completeness sake, we find general expressions for the decay rate and for the Purcell factor that includes contributions of both $\mu_{\perp}^{2}$ and $\mu_{\|}^{2}$ in Eq. 6, averaged over all angles, which gives

$$
\left\langle\mu_{x}^{2}\right\rangle=\left\langle\mu_{y}^{2}\right\rangle=\left\langle\mu_{z}^{2}\right\rangle=\frac{1}{3}\left\langle\mu^{2}\right\rangle
$$

The contribution of each component of the dipole to the decay rate is affected by the presence of the metallic layer and by the dielectric.

Table I. Values of the Purcell factor at various wavelengths for the ion-silver surface distances of $20 \mathrm{~nm}$ and $50 \mathrm{~nm}$ when the dielectric constant of the region 2 (containing the ion) of 1.0 and 2.25 .

\begin{tabular}{lllll}
$d / \varepsilon_{2}$ & $400 \mathrm{~nm}$ & $500 \mathrm{~nm}$ & $600 \mathrm{~nm}$ & $700 \mathrm{~nm}$ \\
\hline $20 \mathrm{~nm} / 1.0$ & 1.75 & 1.37 & 1.28 & 1.22 \\
$50 \mathrm{~nm} / 1.0$ & 1.39 & 1.28 & 1.23 & 1.20 \\
$20 \mathrm{~nm} / 2.25$ & 5.29 & 1.91 & 1.63 & 1.51 \\
$50 \mathrm{~nm} / 2.25$ & 4.13 & 1.70 & 1.53 & 1.45
\end{tabular}


The dipole component parallel to the surface include a factor $\varepsilon_{2} / 2 \varepsilon_{1}$, as seen in Eq. 9. Including the contributions of all the dipoles, Eq. 9 can be rewritten as

$$
B_{s p}=\frac{2 \pi}{\hbar^{2}}\left(\frac{1}{3}\right)\left(1-\frac{\varepsilon_{2}}{\varepsilon_{1}}\right)\left\langle\mu^{2}\right\rangle
$$

We note that $-\varepsilon_{2} / \varepsilon_{1}$ is typically positive and usually less than 1 , so that the perpendicular component is the main contributor to the decay of the ion via emitting a plasmon.

The decay rate due to the presence of the surface plasmon modes is given by inserting Eq. 15 into Eq. 10,

$$
\frac{1}{\tau_{s p}}=\left(\frac{64 \pi^{5}}{3 h c^{3}} \nu^{3}\right)\left\langle\mu^{2}\right\rangle\left(1-\frac{\varepsilon_{2}}{\varepsilon_{1}}\right)\left[\frac{\left(-\varepsilon_{1}\right)^{\frac{5}{2}}\left(\varepsilon_{1}\right)^{\frac{1}{2}} \varepsilon_{2}}{\left(\varepsilon_{1}+\varepsilon_{2}\right)^{\frac{5}{2}}\left(\varepsilon_{2}-\varepsilon_{1}\right)}\right] e^{-2 \alpha_{2} d}
$$

The total radiative decay rate is found by considering both vacuum and plasmon decay modes. Given that the Einstein $B$ coefficient for decay into the vacuum modes is

$$
B=\frac{2 \pi}{3 \hbar^{2}}\left\langle\mu^{2}\right\rangle
$$

Inserting Eqs. 15, 16, and 17 into Eq. 12 leads to

$$
\frac{1}{\tau_{\text {rad }}}+\frac{1}{\tau_{s p}}=\frac{64 \pi^{5}}{3 h c^{3}} \nu^{3}\left\langle\mu^{2}\right\rangle\left\{1+\left(\frac{1}{3}\right)\left(1-\frac{\varepsilon_{2}}{\varepsilon_{1}}\right) f\left(\varepsilon_{1}, \varepsilon_{2}\right) e^{-2 \alpha_{2} d}\right\}
$$

where $f\left(\varepsilon_{1}, \varepsilon_{2}\right)$ is defined in Eq. 13. The corresponding Purcell factor when averaged over all angles is

$$
F=1+\left(\frac{\frac{1}{\tau_{s p}}}{\frac{1}{\tau_{r a d}}}\right)=\left\{1+\left(\frac{1}{3}\right)\left(1-\frac{\varepsilon_{2}}{\varepsilon_{1}}\right) f\left(\varepsilon_{1}, \varepsilon_{2}\right) e^{-2 \alpha_{2} d}\right\} .
$$

Comparing Eq. 19 with Eq. 14, we observe that increase in the decay rate for randomly oriented dipoles compared with dipoles oriented perpendicular to the surface differ by the factor $\left(\frac{1}{3}\right)\left(1-\frac{\varepsilon_{2}}{\varepsilon_{1}}\right)$. For a silver surface, this factor varies from 0.45 to 0.58 from the red to the violet ends of the visible region for a silver surface and for $\varepsilon_{2}=2.25$. Thus, for a system with randomly oriented dipoles, the factor that determines the increase in the decay rate due to the surface plasmon modes will be similar to that shown in Figure 1, except that the decay rate increase should be roughly $\frac{1}{2}$ the values shown. For example, randomly oriented dipoles in a dielectric with $\varepsilon_{2}=2.25$ and emitting at $500 \mathrm{~nm}$, Fig. 1 and Eq. 19 indicate that one should expect an increase in the decay rate due to surface plasmons by a factor of about 1.9 and 1.7 for ions $20 \mathrm{~nm}$ and $50 \mathrm{~nm}$ from the surface, respectively. Figure 1 indicates that these factors should be higher at shorter wavelengths and lower at longer wavelengths, and that all factors increase as $\varepsilon_{2}$ increases.

\section{Conclusions}

We have examined the decay of an optically active ion in the vicinity of a flat, smooth metallic surface. An expression for the density of surface plasmon modes accessible to an ion is shown to depend on the surface plasmon frequency and on the distance of the ion from the surface. Using an Einstein $B$ coefficient appropriate to the ion-surface plasmon interaction and the density of surface plasmon modes, we obtain an expression for the decay rate of the ion into the surface plasmon modes. This result shows that the decay rate of the ion into the surface plasmon modes decreases exponentially as the ion-metal surface distance increases. Also, the decay rate is found to depend on the orientation of the dipole at the ion site relative to the surface the decay rate is stronger if the dipole is oriented perpendicular to the surface compared with those dipoles oriented along the surface. The decay rate has an implicit $\omega$ dependence through the permittivities of the metal and of the dielectric containing the optically active ion. This $\omega$ dependence shows the decay rate increases as the wavelength decreases in the visible region, with the decay rate increasing near the plasmon frequency of the metal. For the case of randomly oriented dipoles, calculations show that the Purcell factor in the visible region due to the presence of silver mirror is shown to be only slightly greater than unity at most wavelengths in the visible region for an ion $20 \mathrm{~nm}$ from the metal surface, with that value decreasing exponentially as the ion-metal distance increases. Thus, the decay into surface plasmon modes will only be significant for ions whose distance to the mirror is much less than the wavelength of the photon.

\section{Acknowledgment}

The authors thank Dr. Alan Piquette from OSRAM Sylvania for many helpful discussions.

\section{ORCID}

J. Collins (1D https://orcid.org/0000-0002-9567-1039

K. C. Mishra (1D https://orcid.org/0000-0003-4081-2858

\section{References}

1. K. C. Mishra, J. Collins, and A. Piquette, ECS Journal of Solid State Science and Technology, 7(5) R42 (2018).

2. M.-K. Kwon, J.-Y. Kim, B.-H. Kim, I.-K. Park, C.-Y. Cho, C. C. Byeon, and S.-J. Park, Adv. Mater, 20, 1253 (2008).

3. K. Okamoto, I. Niki, A. Shvartser, G. Maltezos, Y. Narukawa, T. Mukai, Y. Kawakami, and A. Scherer, Phys. Stat. Sol. A, 204, 2103 (2007).

4. K. Okamoto, I. Niki, A. Shvartser, Y. Narukawa, T. Mukai, and A. Scherer, Nat. Mater, 3, 601 (2004).

5. J. Vuckovic, M. Loncar, and A. Scherer, IEEE J. Quantum Electron., 36, 1131 (2000)

6. J. Henson, J. DiMaria, E. Dimakis, T. D. Moustakas, and R. Paiella, Opt. Lett., 37, 79 (2012).

7. J. Henson, J. C. Heckel, E. Dimakis, J. Abell, A. Bhattacharyya, G. Chumanov, T. D. Moustakas, and R. Paiella, Appl. Phys. Lett., 95, 151109 (2009).

8. I. Gontijo, M. Boroditsky, E. Yablonovitch, S. Keller, U. K. Mishra, and S. P. DenBaars, Phys. Rev. B, 60, 11564 (1999).

9. N. E. Hecker, R. A. Höpfel, and N. Sawaki, Physica E, 2, 98 (1998).

10. A. Neogi, C.-W Lee, H. O. Everitt, T. Kuroda, A. Tackeuchi, and E. Yablonovitch, Phys. Rev. B, 66, 153305 (2002).

11. G. Lozano, S. R. K. Rodriguez, M. A. Verschuuren, and J. G. Rivas, Light Sci. Appl., 5, e16080 (2016).

12. W. H. Chao, R.-J. Wu, C.-S. Tsai, and T.-B. Wu, J. Appl. Phys., 107, 013101 (2010).

13. S. M. Lee and K. C. Choi, Opt. Express, 18, 12144 (2010).

14. E. Cohen-Hoshen, G. W. Bryant, I. Pinkas, J. Sperling, and I. Bar-Joseph, Nano Lett., 12, 4260 (2012).

15. K. Munechika, Y. Chen, A. F. Tillack, A. P. Kulkarni, I. Jen-La Plante, A. M. Munro, and D. S. Ginger, Nano Lett., 11, 2725 (2011).

16. H. Mertens, J. S. Biteen, H. A. Atwater, and A. Polman, Nano Lett., 6, 2623 (2006).

17. A. Gopinath, S. V. Boriskina, S. Yerci, R. Li, and L. Dal Negro, Appl. Phys, Lett., 96, 071113 (2010).

18. C. Jang, S. M. Lee, and K. C. Choi, Opt. Express, 20, 2143 (2012).

19. H. Mertens and A. Polman, Appl. Phys. Lett., 89, 211107 (2006).

20. L. Xu, Y. Qiang, K. Xiao, Y. Zhang, J. Xie, C. Cui, P. Lin, P. Wang, X. Yu, F. Wu, and D. Yang, Appl. Phys. Lett., 110, 233113 (2017)

21. W. Ye, Q. Huang, X. Jiao, X. Liu, and G. Hu, J. Alloy. Compd., 719, 159 (2017).

22. O. L. Muskens and J. Gómez -Rivas, Mater. Sci. Eng. B, 149, 216 (2008).

23. A. Nikitin, M. Remezani, and J. Gómez-Rivas, ECS J. Solid State Sci. Technol., 5, R3164 (2016)

24. E. M. Purcell, Phys. Rev., 69, 681 (1946).

25. K. H. Drexhage, J. Luminescence, 1, 2, 693 (1970).

26. K. H. Drexhage, "Interaction of Light with Monomolecular Dye Layers", Progress in Optics, E. Wolf, ed., North Holland Press, 1974.

27. A. Archambault, F. Marquier, J.-J. Greffet, and C. Arnold, Phys. Rev. B, 82035411 (2010).

28. D. P. Craig and T. Thirunamachandran, Molecular Quantum Electrodynamics, An Introduction to Radiative Molecule Interactins, Dover Publications, New York, 1984

29. H. Yang, J. Archangel, M. Sundheimer, E. Tucker, G. Boreman, and M. Raschke, Phys. Rev., 91, 235137 (2015). 